Revue scientifique francophone en Communication

organisationnelle

$18 \mid 2000$

Non-verbal, communication, organisation

\title{
La communication non-verbale dans l'entretien de sélection
}

Abdelouahed Noukoud

\section{OpenEdition}

Journals

Édition électronique

URL : https://journals.openedition.org/communicationorganisation/2423

DOI : 10.4000/communicationorganisation.2423

ISSN : $1775-3546$

\section{Éditeur}

Presses universitaires de Bordeaux

\section{Édition imprimée}

Date de publication : 1 novembre 2000

ISSN : 1168-5549

Référence électronique

Abdelouahed Noukoud, "La communication non-verbale dans l'entretien de sélection »,

Communication et organisation [En ligne], 18 |2000, mis en ligne le 27 mars 2012, consulté le 05 août

2021. URL : http://journals.openedition.org/communicationorganisation/2423 ; DOI : https://doi.org/

10.4000/communicationorganisation.2423

Ce document a été généré automatiquement le 5 août 2021.

(C) Presses universitaires de Bordeaux 


\title{
La communication non-verbale dans l'entretien de sélection
}

\author{
Abdelouahed Noukoud
}

\section{Introduction}

1 L'objet de cet article est de mettre l'accent sur la dimension de la communication nonverbale dans l'entretien de sélection.

2 L'entretien de sélection se caractérise à la fois par une interaction verbale et nonverbale entre le recruteur et le candidat, il a pour but de rechercher des compétences, mais aussi d'avoir des impressions et de recueillir des informations qui corroborent la réalité du candidat au profil recherché.

3 La relation duelle dans cet entretien revêt des intentions et des comportements psycho-cognitifs dont les effets suscitent l'activation du processus interprétatif des interactants.

4 D'une part, le candidat à la sélection se met en scène dans le cadre de l'entretien, d'autre part, le recruteur décode, filtre les messages reçus, confronte ensuite les informations significatives au profil recherché. De par sa représentation sociale et sa position dans la situation de l'entretien de sélection, le recruteur va se positionner par rapport à une valeur socialement définie «le profil recherché ».

5 Évidemment, à l'issue de l'entretien, le recruteur est amené à faire un choix. Il ne peut le faire qu'à l'aide d'une lecture interprétative de la communication verbale et nonverbale. Ce processus interprétatif des mots et de signes, est en quelque sorte un système d'évaluation, pour deux raisons :

$6 \quad$ 1) il gère la complexité de la sélection face à la diversité et la singularité des candidats.

7 2) il prépare et facilite la prise de décision de sélection, tout autant au niveau de la logique rationnelle qu'au niveau de la logique intuitive.

8 Malgré les moyens de traitement du langage verbal dans l'entretien de sélection, l'information obtenue est insuffisante pour rendre compte de l'efficacité de la décision. 
C'est pourquoi la communication non-verbale dans l'entretien de sélection est d'une importance telle qu'elle lui donne toute la consistance théorique et pratique dans la détermination du choix du candidat idéal. Dès maintenant, nous pouvons déjà émettre presque un théorème :

10 Plus la communication non-verbale est intense dans la situation duelle de l'entretien de sélection, plus les stratégies de persuasion et de séduction mises en œuvre par le candidat, jouent un rôle déterminant dans l'information et la formation de l'impression du recruteur. Inversement, plus cette dimension est insignifiante d'interactions, moins probables seront ses effets sur la décision de sélection.

11 Nous nous contentons d'étudier, ici, l'activité du recruteur dans le cadre de l'entretien de sélection pour des raisons qui tiennent à la délimitation de l'objet de notre recherche.

«Les hommes ne parlent pas seulement avec des mots, mais aussi avec leur corps,

avec les objets, avec leur organisation de l'espace et du temps $»^{1}$.

12 En effet, le corps humain est non seulement un support de sensations et d'émotions, mais il est aussi porteur de significations et de sens. La communication non-verbale est la dimension cachée dans toute interaction, car dans la réalité de la sélection tout est important : le vocal, le visuel, le postural, le gestuel... Tous ces marqueurs sociaux sont autant de facteurs qui informent sur la personne du candidat, sur son état psychique et moral, sur son état de santé, sur son humeur, sur sa vigilance...

«Ce langage silencieux présente certains traits du verbal, et notamment celui d'être un code en partie arbitraire, différent d'une culture à l'autre $»^{2}$.

13 Aussi paradoxal que cela puisse paraître, le recruteur traite parallèlement, logiquement et intuitivement les informations et les signes de la communication non-verbale, dans l'entretien de sélection, sous forme de grilles de lecture sémiologique. Or, cela suppose l'application de nouvelles approches pour en décrypter les messages et en décoder les signes. Ceci afin d'investir, peu à peu, les significations de l'irrationnel, à la recherche d'une validation scientifique des méthodes de recrutement, dans l'approche des candidats à la sélection.

\section{L'information dans l'interaction non-verbale de l'entretien de sélection}

Lorsqu'un candidat est convoqué pour l'entretien de sélection et mis en situation de communication, l'interaction s'installe sous forme dialectique entre les partenaires de l'échange dans le cadre spécifique de cet entretien.

C'est parce qu'il s'agit de faire un choix entre les candidats, que l'activité psychocognitive du recruteur doit prendre acte, par un travail de repérage et d'identification de différentes formes et contenus de la communication. Il va de soi que la finalité de cette activité est de mobiliser les renseignements, en terme d'information pertinente, au sujet des candidats à la sélection. Cette information doit être traitée, interprétée, évaluée, voire même stockée en cas de besoin. Ainsi le recruteur sera plus à même de gérer, en amont, le choix et réduire au mieux toute la complexité et l'incertitude qu'il comporte. 

communication verbale, a pour objet l'étude des impressions et des expressions corporelles du point de vue de la forme et des signes (la sémiologie, la rhétorique). communication non-verbale et la pertinence de l'information quelle procure pour diverses raisons :

18 - la littérature sur la communication non-verbale ne s'intéresse pas assez aux situations professionnelles (peu d'écrits à ce sujet),

19 - l'approche sémiologique, de l'interaction dans la situation de l'entretien de sélection, peut se révéler efficace dans le processus d'évaluation des candidats.

20 - les expressions corporelles qu'émettent les individus et les images qu'ils renvoient, dans les situations d'interaction, échappent pour une grande part à leur contrôle.

21 - l'information obtenue se prête facilement à l'interprétation, malgré l'augmentation de son volume, elle peut se révéler riche de significations.

22 - la confrontation des informations issues des deux dimensions de la communication permet, à juste titre, le repérage des paradoxes, des conflits psycho-cognitifs des candidats et informe sur leur personnalité.

\section{Le constat par rapport à l'information}

Dire que l'information, dans l'interaction, n'a de sens qu'à travers celui qui peut en rendre compte, c'est poser, en fait, la question sur les sources de sa production.

"Notre perception s'appuie sur les indices verbaux pour un tiers seulement des messages échangés, les deux autres tiers s'établissent à partir des indices non verbaux $»^{3}$.

En somme, la part de l'information tirée du non-verbal est révélatrice d'un autre niveau d'information, plus pertinent ou, tout au moins complémentaire dans l'investigation de la vérité.

«On ne peut pas ne pas communiquer, qu'on le veuille ou non. Activité ou inactivité, parole ou silence, tout a valeur de message $»^{4}$

Or, l'expérience montre que le candidat, dans la situation de l'entretien, peut difficilement contrôler ses expressions corporelles. La production des comportements irrationnels n'obéit ni à la raison, ni à son contrôle. Ce ne sont pas des actes isolés, sans relation avec l'échange verbal en particulier, et le réel du candidat de façon générale.

«On ne peut saisir en effet des attitudes, des croyances et des émotions vraies ou réelles chez quelqu'un qu'à travers ses aveux ou, de façon indirecte, à travers ce qui apparait comme comportement involontairement expressif $»^{5}$.

27 Ainsi, l'analyse de la communication non-verbale dans les situations professionnelles se révèle en soi d'un intérêt crucial quant aux niveaux d'information qu'elle suscite et aux méthodes d'investigation qu'elle interpelle. Mais pour saisir la richesse des informations, les recruteurs doivent utiliser les méthodes scientifiques vérificatives et les outils adéquats à son analyse. 


\section{La démarche sémiologique de l'implicite dans l'entretien de sélection}

Pour qu'elle soit optimisée, la communication doit convoquer la méthode sémiologique. étant donné que celle-ci a pour objet l'étude des signes et leur système de fonctionnement. Sa consistance réside en ce qu'elle permet de percevoir les atouts et les dysfonctionnements des interactions dans les situations de communication.

Raison de plus pour les recruteurs de se pencher sérieusement sur cette méthode, car l'obtention d'informations relatives à la personnalité du candidat, à ses contradictions, à son univers de référence, à sa compétence, va au-delà du contenu de l'échange verbal et de son argumentaire. Le décryptage, a priori insignifiant, qu'opère la sémiologie des messages visuels de l'interaction, dans la relation duelle de l'entretien, fait sens au fur et à mesure que l'analyse s'affine en corrélation du dit et du non dit. La lecture connotative des signes dans l'interaction devrait, en principe, informer sur les formes et les contextes de la dénotation des messages dans l'entretien de sélection.

Appliquée à l'entretien de sélection, la sémiologie met en évidence les signes, les indices, les images, les postures, les couleurs, l'habillement et les façons d'être, en résumé, toutes les expressions et impressions corporelles des partenaires de l'échange dans l'entretien.

\section{La réception des impressions : un rôle déterminant dans la sélection}

31 L'impression dans l'entretien de sélection est l'image que le candidat laisse sur le recruteur, via ses expressions verbales et corporelles, pendant l'interaction.

Dans la situation de l'entretien, faut-il se demander comment se forment les impressions des recruteurs? Obéissent-elles à des critères objectifs ou à des critères subjectifs? Et enfin, quel rôle jouent-elles dans le processus de la décision de la sélection?

La construction de l'image du candidat se fait tout au long des procédures de sélection, l'apparence et l'image communiquées pendant l'entretien par le candidat, non seulement elles précèdent et accompagnent sa parole, mais leur effet reste imprégné dans la mémoire du recruteur. De toute façon, les expressions corporelles du premier interviendront obligatoirement dans l'information et la formation de l'impression du second, et par conséquent elles s'avèrent décisives dans le processus de sélection. Nous tenons à donner un exemple tout à fait significatif quant au niveau des impressions, lors d'un entretien de sélection ${ }^{6}$ auquel nous avons assisté.

Les séquences de cet entretien :

Un membre du jury sort du lieu de l'entretien pour accueillir une candidate. La candidate mâchait du chewing-gum. Surprise par son arrivée, elle le jeta à la poubelle. L'interaction commence, la sonorité de sa voix paraît grave. Après l'entretien, les membres du jury échangèrent leurs conclusions. Un membre du jury "non-fumeur » argumente son impression: elle a la voix grave, parce qu'elle fume, c'est pour cette raison qu'elle mâchait du chewing-gum, car elle voulait cacher l'odeur de la cigarette, ça m'a fait une mauvaise impression. Nous avons su par la suite que cette candidate ne figurait pas dans la liste des admis à la formation. 
«Cette rémanence. parfois plus durable que les mots crédite ou discrédite notre propos... $»^{7}$.

«Dans la vie quotidienne en effet, les premières impressions sont fondamentales " $^{8}$. Si l'impression est bonne, dans ce cas, ceci plaidera en la faveur du candidat, si elle est mauvaise, elle le discrédite. Finalement l'impression ne peut être que la réception des expressions verbales et corporelles au cours d'une situation d'interaction.

\section{L'émission des expressions corporelles à l'examen de l'entretien de sélection}

Certaines modalités corporelles (regard, gestes, voix, postures, vêtements...), peuvent réguler, compléter ou contredire le verbal dans la communication. Ces modalités devraient permettre aux recruteurs qui gèrent ou passent l'entretien, de mieux analyser et parfois contrôler certaines variables qui influencent le déroulement des interactions dans l'entretien.

\section{Le regard : une source d'informations pertinentes dans l'interaction}

L'œil est un organe de sens performant dans l'acuité visuelle, mais il est aussi un moyen de communication, son importance réside en ce qu'il est à la fois émetteur et récepteur de messages en terme de regard.

regard est l'une des sources d'inspiration pour les écrivains et les poètes : « il y a des regards dans ton regard » disait un romancier à sa bien-aimée, le regard est aussi une source d'information importante pour les psychologues, sémiologues et recruteurs. Dans la mesure où le regard est le lieu de production de signes de communication.

Dès l'interaction, de l'entretien de sélection, le recruteur établit un contact visuel permanent avec le candidat. Ce contact contribue non seulement à renforcer la relation entre candidat et recruteur au plus intime, mais aussi à traduire un sentiment réconfortant de «bon signe " chez le candidat. Une fois que l'expression visuelle est émise, elle est machinalement, entièrement ou partiellement perçue par le recruteur.

«Le regard d'une personne contribue, tout autant que les mots, à son charisme personnel ou, à l'inverse, s'il est fuyant, lui fait perdre un certain crédit »".

41 Si le regard n'exprime pas exactement les mots, il exprime les choses et les signes. Dans ce cas, quels sont les critères de validité de la qualité du regard?

Le regard peut exprimer diverses situations psychoaffectives telles que l'amour, la peur, l'angoisse, l'anxiété, la sincérité, la confiance en soi, etc. Reste à savoir que l'expression du regard est souvent actualisée par le geste et les mouvements du corps, une sorte d'accord dont la moindre fausse note suscite bien des interrogations et des interprétations du recruteur.

\section{Les gestes et postures : des indicateurs puissants dans la relation de l'entretien}

Si le geste a précédé la parole, ne fonde-t-il pas la communication autant que le verbe, dans les situations d'interaction les plus diverses et, en particulier dans l'entretien de sélection? Les recruteurs ne devraient-ils pas s'intéresser aux signes de ce langage silencieux de la communication?

Communication et organisation, 18 | 2000 
En effet, prêter plus d'attention à la question des expressions corporelles du candidat, dans le contexte de l'entretien, permet d'en vérifier les codes, les signes et les messages de la communication et d'en qualifier leur teneur par rapport à la réalité de la sélection.

"C'est pourquoi d'ailleurs un signe est rarement isolé ; il se situe dans le contexte d'autres signes auxquels il est associé, et dont il tire sa signification, d'autant plus que le même geste peut avoir plusieurs sens» ${ }^{10}$

Or, le candidat, dans l'entretien, peut préméditer certains gestes et postures, dans un but purement stratégique, afin de retenir l'attention du recruteur. Il essayera au cours de l'interaction d'optimiser sa mimogestualité, son regard, son argumentation pour convaincre, voire même séduire le recruteur.

maitrise par certains candidats des techniques connues de la communication leurs gestes et leur regard finiront par les trahir, à un moment ou à un autre de l'entretien. Car certaines expressions du corps sont surtout une production de l'inconscient, donc difficile à contrôler dans ce genre de situations, justement parce qu'involontaires.

7 Des études à ce sujet montrent bien la relation étroite entre la manifestation de nos émotions et sentiments et les gestes qui les matérialisent.

8 Le geste se lit selon les messages émis et s'interprète selon un code, plus au moins formalisé, plus au moins universel. Nous pensons que la relation entre le geste et sa signification n'a de sens que dans le contexte culturel de sa production. L'universalité $\mathrm{du}$ geste et des expressions corporelles, est une conclusion hâtive, car un universalisme gestuel conduirait automatiquement à une exclusion du particularisme gestuel relatif à chaque culture. Cela se traduirait, dans les faits, par une fausse interprétation des expressions corporelles des différents candidats et, donc, une discrimination de la sélection. D'autant plus que le recrutement s'annonce mondial.

\section{La voix et les hésitations dans l'interaction : un signe révélateur}

9 La voix du candidat dans l'interaction de l'entretien joue autant que ce qui va être dit, la tonalité de la voix l'emporte souvent sur le contenu du message. En effet, tout le monde est d'accord que le timbre d'une voix agréable met l'interlocuteur à l'aise, mais en même temps la voix assure la mise en valeur du contenu de l'acte langagier. Elle est le support de la parole, en ce sens elle permet de mieux présenter le candidat dans l'entretien de sélection.

«La vocalité qui joue un rôle capital pour la réalisation des fonctions expressives et esthétiques $»^{11}$.

50 Pour quelles soient optimisées, aux yeux du recruteur, les expressions corporelles, doivent être médiatisées dans l'entretien, par l'habit qui lui donne la forme la plus adéquate au profil recherché.

\section{Le vestimentaire : un atout de valorisation}

51 Dans la vie de tous les jours, les gens accordent plus d'importance à l'apparence, à l'image qu'ils donnent d'eux. Celle-ci détermine a priori leur valeur sociale aux yeux d'autrui. Si cela est vrai, alors quels messages peut exprimer le vestimentaire dans le 
cadre de l'entretien de sélection? Et quels impacts cela produit-il sur le recruteur dans le processus d'interprétation de signes?

La ritualité protocolaire propre aux organisations exige que l'entretien soit en bonne et due forme, cela veut dire que les partenaires de l'interaction doivent être habillés de manière à exprimer fidèlement leurs rôles sociaux respectifs.

$\mathrm{Au}$ fond, c'est cette mise en scène, dans tous ses aspects, qui fonde le positionnement social du candidat et du recruteur par rapport au poste à pourvoir. La façon dont le prétendant est habillé exprime en grande partie sa réalité, elle informe, avec une forte probabilité, sur son milieu social, ses goûts et sur son caractère. "Le vêtement fait le lien entre l'être et le paraître, entre les sphères $d u$ « soi » et du « moi $»^{12}$... Mais pour entrevoir l'exactitude de ces informations, il faut que le recruteur passe au peigne fin plusieurs variables vestimentaires : ainsi le choix des couleurs, la coupe et la matière du vêtement, la cravate... Ces variables ont un effet immédiat sur l'impression du recruteur, elles doivent être analysées au-delà du contexte de l'entretien.

«Le vêtement est un véritable canal de communication sociale..., c'est une prolongation de la personnalité de l'individu..., un véritable langage socio-culturel dont il resterait à définir plus précisément les codes.... ${ }^{13}$

C'est pour cette raison que le choix de l'habit devrait être confronté, implicitement par le recruteur en situation, au profil recherché, et établir une sorte de projection du candidat dans l'univers des signes, par rapport au poste qu'il est susceptible d'occuper.

\section{Conclusion}

5 L'analyse de la communication non-verbale, dans le contexte de l'entretien de sélection, donne lieu à un second niveau d'information, différent de l'information obtenue à partir des actes verbaux.

6 Dans l'entretien de sélection, l'emploi de nouvelles approches telles que la sémiologie s'avère fécond, celle-ci devrait fournir aux recruteurs une lecture pertinente des signes produits pendant l'interaction. Ainsi, sous le regard sémiologique du recruteur, les expressions corporelles et langagières des candidats, prennent un autre sens, parce que la sémiologie agit comme une loupe sous laquelle les facettes de la personnalité se dessinent à grands traits. Étant donné que le signifiant véhicule le signifié.

L'intérêt de cette approche c'est de mettre en relation les signes et leurs oppositions : par exemple baisser les yeux n'est signifiant que parce qu'il y a embarras du candidat par rapport à une question dans l'interaction. La sémiologie permet en outre la relation entre les signifiants et le signifié, car faire abstraction de cette relation dans l'entretien conduirait forcement à un manque de pertinence de l'information au sujet du candidat à la sélection.

Finalement, se résoudre à une telle démarche, dans l'entretien de sélection, c'est admettre davantage la vérification de l'adéquation des signes de la communication non-verbale au sens de ce qui est dit par le candidat pendant l'interaction de l'entretien de sélection. 


\section{BIBLIOGRAPHIE}

BARRIER G., La communication non-verbale, Aspects pragmatiques et gestuels des interactions, ESF éditeur, Paris, 1996, $156 \mathrm{p}$.

BITOUN C, MAIER C, « La sémiologie au service de la prévision en analyse industrielle », Revue française du marketing. $\mathrm{n}^{\circ}$ 175, 1999/5 Bonu B., « Perturbation et négociation dans l'entretien de recrutement », Langage et société nº 89. septembre 1999.

COSNIER J., BROSSARD A., CONDON W.S., COOK M., CRAWFORD R., DAW W., EKMAN P., FLORIN A.M., FRIESEN W., FREY S., HIRSBRUNNER H.P., SCHERER R.K., La communication non-verbale, Delachaux et Niestlé, Neuchâtel, Paris. 1992, 244 p.

GOFFMAN E., La mise en scène de la vie quotidienne, la présentation de soi, Les éditions de minuit, Paris 1973, $251 \mathrm{p}$.

GUIRAUD P., Le langage du corps, que sais-je ? Puf. nº 1850, Paris. 1980, 127 p.

HALL T. E., La Dimension cachée, Paris, Éditions du Seuil, 1971, 256 p. Langage silencieux. Éditions Mame Tour-1973, 223 p.

DURAND J., Les formes de la communication, BORDAS, Paris, 1981, p. 5-6.

SIEGEL S.M., FRIEDLANDER M.L., HEATHERINGTON L. « Nonverbal relational control in family communication ", Journal of Nonverbal Behavior, 16, 117-139 (1992)

VAN CUYCK A., " Le vêtement canal social de communication ", note de recherches ERSICO, mars 1992.

\section{NOTES}

1. Durand J., Les formes de la communication, Bordas, Paris, 1981, p.5-6.

2. Corraze J., cité par Jacques Durand, Les formes de la communication.

3. Siegel S.M., Friedlander M.L., Heatherington L., « Non verbal relational control in family communication ». Journal of Nonverbal Behavior, 16, 117-139 (1992).

4. Watzlawick P., Helmic-Beavin J.. Jackson D., Une logique de la communication, Paris Ed. du Seuil, p.46.

5. Goffman E., La mise en scène de la vie quotidienne, la présentation de soi, p. 12.

6. Il s'agit d'un entretien de sélection des étudiants pour intégrer la filière d.e.s.s inl'o-com, Lyon III.

7. Barrier G., La comunication non verbale, p. 13.

8. Goffman E., La mise en scène de la vie quotidienne, la présentation de soi, p.9.

9. Barrier G., La communication non verbale, p.49.

10. Guiraud P., Le langage du corps. Que sais-je ?, n 1850, p.32.

11. Cosnier J. et Brossard A., La communication non verbale, p.5.

12. Van Cuyck A., "Le vêtement canal social de communication », note de recherches ERSICO, Mars 1992, p.4.

13. op.cit., p. 10, Van Cuyck A. 


\section{AUTEUR}

ABDELOUAHED NOUKOUD

Doctorant, Université Jean Moulin-Lyon III (ERSICO) 